\title{
Semidirect Products of Association Schemes
}

\author{
SEJEONG BANG* \\ sjbang3@ postech.ac.kr \\ Combinatorial and Computational Mathematics Center, Pohang University of Science and Technology, Pohang \\ 790-784, Korea
}

MITSUGU HIRASAKA

hirasaka@pusan.ac.kr

Department of Mathematics, Pusan National University, Pusan 609-735, Korea

SUNG-YELL SONG*

Department of Mathematics, Iowa State University, Ames, Iowa, 50011, USA

sysong@iastate.edu

Received March 9, 2001; Revised January 12, 2004; Accepted February 9, 2004

\begin{abstract}
In his 1996 work developing the theory of association schemes as a 'generalized' group theory, Zieschang introduced the concept of the semidirect product as a possible product operation of certain association schemes. In this paper we extend the semidirect product operation into the entire set of association schemes. We then derive a way to decompose certain association schemes into smaller association schemes. We also investigate to what extent this product helps us to understand and characterize the structure of association schemes. We give some examples to show that the semidirect product produces many schemes that cannot be described as neither the direct product nor the wreath product of smaller schemes.
\end{abstract}

Keywords: semidirect product, association scheme

\section{Introduction}

There are many ways to construct new association schemes from old ones. Association schemes can be built up from 'smaller' ones; two important constructive methods are the direct product and wreath product. Another way to construct new association schemes from old is by fusion and fission processes - the processes in which a new association scheme is obtained by combining or splitting relations of the old scheme in a certain way (cf. [8]). In this paper, the semidirect product operation is introduced as yet another way to construct new association schemes from smaller ones.

In 1996, Zieschang introduced a new product operation of an association scheme with a group acting on the scheme: the semidirect product. By 'a group acting on a scheme' we mean that there is a (group) homomorphism from the group to the (combinatorial) automorphism group of the association scheme (cf. [10], Section 2.7). In 2000, Muzychuck generalized the product operation by using 'a group acting on the set of association relations'; i.e., employing a homomorphism from the group to the 'algebraic automorphism group' of the association scheme (cf. [6]). By using the Muzychuk's operation, we can produce some 
other association schemes in addition to those that can be obtained from the Zieschang's operation. However, both operations are restricted to taking the product of an association scheme with a 'thin' association scheme. They are not defined to operate for a pair of arbitrary association schemes; one of the factors of the product is required to be a thin association scheme. The 'semidirect product' which will be defined in Section 3 extends Muzychuk's operation so that it can operate on the entire set of association schemes as a natural extension of both Zieschang and Muzychuk's operations.

The semidirect product produces many imprimitive association schemes. The class of primitive schemes in the theory of association schemes plays a role like that of simple groups in group theory (cf. [2, 3, 10]). As far as the classification problem of association schemes is concerned, imprimitive schemes are as important as primitive schemes. There are two practical reasons for this. First, imprimitive schemes arise in many parts of combinatorics such as graph theory and design theory. Second, many primitive schemes can be obtained as fusion schemes of imprimitive schemes. Our aim is to see to what extent the semidirect product, together with fusion and fission processes, can produce new imprimitive schemes and distinguish them from other known association schemes.

In recent years, there was a successful attempt to obtain the complete list of isomorphism classes of all association schemes of given order $n$, for $n$ up to 32 (See, [4, 8], for example). However, for the class of association schemes with larger orders, the search to find all the association schemes becomes increasingly complicated. Therefore, we have been searching for more tools to construct and describe association schemes from a relatively small set of association schemes. The current paper is expected to make some contribution to the research in this direction.

This paper is organized as follows. In Section 2, we set up the notation and terminology, and recall some basic facts about association schemes. In Section 3, we construct the external and internal semidirect products of association schemes; so, we have a decomposition theorem of certain association schemes into factors. We give some examples of association schemes characterized as semidirect products of certain association schemes. In Section 4, we show an application of the product in the classification problem and structure theory of association schemes. In particular, we give a characterization of a few association schemes that are not decomposed by direct or wreath products. The section closes with a few remarks in regard to the product operations and the classification of association schemes.

\section{Preliminary facts and notation}

We begin this section by recalling the definition of an association scheme following the notation of [10]. Let $X$ be a finite set of points. We denote the diagonal relation $\{(x, x) \mid x \in$ $X$ \} on $X$ by $1_{X}$ (or by 1 when there is no risk of confusion). For an arbitrary relation $a \subset X \times X$ and a point $z \in X$, we let

$$
a^{*}:=\{(x, y) \mid(y, x) \in a\} \text { and } z a:=\{x \in X \mid(z, x) \in a\}
$$

for the transpose of $a$ and the set of points being in relation $a$ with $z$, respectively. 
Definition 2.1 Let $H$ be a partition of $X \times X$ with non-empty relations on $X$. We call $(X, H)$ an association scheme (or simply, a scheme) if it satisfies the following conditions:

(i) $1 \in H$;

(ii) $a^{*} \in H$, for each $a \in H$;

(iii) For any $a, b, c \in H$, the number $\left|x a \cap y b^{*}\right|$ is constant for every $(x, y) \in c$. i.e., it depends only on $a, b, c$.

The cardinality $\left|x a \cap y b^{*}\right|$ of the set $x a \cap y b^{*}$ for $(x, y) \in c$ is denoted by $p_{a b}^{c}$, and is called an intersection number of $(X, H)$. For each relation $a \in H$, the intersection number $p_{a a^{*}}^{1}=|x a|$ is called the valency of $a$. If the valency of a relation $a \in H$ is one, then the relation is called a thin relation. If all relations of a scheme are thin, then the scheme is called thin. For each pair of points $(x, y) \in X \times X$, the unique relation of $H$ which contains $(x, y)$ is denoted by $r(x, y)$.

Definition 2.2 Let $(X, H)$ and $(Y, K)$ be two schemes. We say that $(X, H)$ is isomorphic to $(Y, K)$, denoted by $(X, H) \simeq(Y, K)$, if there exists a bijection

$$
\theta: X \cup H \rightarrow Y \cup K
$$

such that

$$
\theta(X)=Y, \quad \theta(H)=K, \quad \text { and } \quad \theta(r(x, y))=r(\theta(x), \theta(y))
$$

for all $x, y \in X$. The set of all isomorphisms from $(X, H)$ to itself is denoted by $\operatorname{Aut}(X, H)$ and is called the (weak combinatorial) automorphism group of the scheme.

We also recall the 'algebraic automorphism group' which will be used throughout. For given a set $H$, let $\operatorname{Sym}(H)$ denote the symmetric group on $H$. We write permutations on the shoulder, that is, the image of $a$ under the permutation $\sigma$ is $a^{\sigma}$. We compose from left to right so that $\left(a^{\sigma}\right)^{\pi}=a^{\sigma \pi}$. We may suppose that $H=\{0,1, \ldots, n-1\}$ if $|H|=n$, and that we are dealing with elements of the symmetric group $S_{n}$ instead of those of $\operatorname{Sym}(H)$ at times. The identity permutation will be denoted by $\iota$ throughout.

Definition 2.3 Let $(X, H)$ be an association scheme, and set

$$
\operatorname{Aut}(H):=\left\{\sigma \in \operatorname{Sym}(H) \mid p_{a b}^{c}=p_{a^{\sigma} b^{\sigma}}^{c^{\sigma}}, \text { for all } a, b, c \in H\right\} .
$$

Then, $\operatorname{Aut}(H)$ is a subgroup of $\operatorname{Sym}(H)$. $\operatorname{Aut}(H)$ is called the algebraic automorphism group of $(X, H)$.

We note that each element $\sigma$ of $\operatorname{Aut}(H)$ satisfies that

$$
1^{\sigma}=1 \quad \text { and } \quad\left(a^{*}\right)^{\sigma}=\left(a^{\sigma}\right)^{*} \quad \text { for each } a \in H .
$$

The terms 'combinatorial' and 'algebraic' automorphism groups are due to [7]. 
For each subset $E \subseteq H$, we let $E^{*}:=\left\{a^{*} \mid a \in E\right\}$. Given $x \in X, x E$ denotes the union of sets $x a$ over all $a \in E$. Following [10], we define the 'complex product' as a binary operation on the power set of the relation set $H$.

Definition 2.4 Let $(X, H)$ be an association scheme. The complex product of two subsets $E, F$ of $H$ is defined by

$$
E F:=\bigcup_{z \in X}\left\{r(x, y) \mid x \in z E^{*} \text { and } y \in z F\right\}=\left\{h \in H \mid \sum_{e \in E, f \in F} p_{e f}^{h} \neq 0\right\}
$$

Given $\{e\},\{f\}, F \subseteq H$, for the notational simplicity, we denote the complex product $\{e\} F$ by $e F$, and that of $\{e\}$ and $\{f\}$ by $e f$. So, we have

$$
e f=\left\{h \in H \mid p_{e f}^{h} \neq 0\right\}
$$

From the definition of the complex product, it is clear that: for every $e, f, g \in H$,

(i) $e \in f g$ if and only if $f \in e g^{*}$.

(ii) $1 \in e^{*} f$ if and only if $e=f$.

It is also clear that $(X, H)$ is thin if and only if $h h^{*}=\{1\}$ for each $h \in H$.

Definition 2.5 A nonempty subset $F \subseteq H$ is called closed if

$$
F F^{*} \subseteq F
$$

or equivalently, if $\bigcup_{b \in F} b$ is an equivalence relation on $X$.

It follows from the definition that $\{1\}$ and $H$ are trivial and improper closed subsets of $H$, respectively. Since 1 belongs to $h h^{*}$ for any $h \in H, 1$ belongs to every closed subset of $F$. This, in turn, implies that $F^{*}=F$, and thus $F F^{*}=F F=F^{*} F \subseteq F$ for any closed subset $F$ of $H$. Furthermore, given closed subsets $E, F$ of $H$, we can see that

$$
E F \text { is closed if and only if } E F=F E \text {. }
$$

Thin schemes can be treated like groups in many instances. In [10, p. 39], the 'thin residue' of a closed set $F$ is introduced in the process of constructing factor schemes that are thin. The semidirect product will be constructed via factor schemes of thin residues. Throughout, the notation $E \leq F$ will be used if $E \subseteq F$ and both $E, F$ are closed subsets of $H$. For $E \subseteq H$, the smallest closed subset of $H$ containing $E$ will be denoted by $\langle E\rangle$. We now define the thin residue of $F$ as follows. 
Definition 2.6 Let $(X, H)$ be an association scheme, $F \leq H$, and let

$$
\mathbf{O}^{\vartheta}(F):=\left\langle\bigcup_{f \in F} f f^{*}\right\rangle .
$$

The closed subset $\mathbf{O}^{\vartheta}(F)$ of $F$ is called the thin residue of $F$.

This definition is equivalent to the one given as the intersection of the 'strongly normal' closed subsets of $F$ (cf. [10, Theorem 2.3.1]). The thin residue $\mathbf{O}^{\vartheta}(H)$ of $H$, which will be used a lot in what follows, is the uniquely determined smallest closed subset of $H$ the factor scheme of which is thin. First, we recall the definitions of subschemes and factor schemes.

Definition 2.7 Let $(X, H)$ be an association scheme. Given a closed subset $F \subseteq H$ and a point $x \in X$, set $S=x F$ and let

$$
(X, H)_{S}:=\left(S,\left\{f_{S}\right\}_{f \in F}\right), \quad \text { where } f_{S}:=f \cap(S \times S) .
$$

Then $(X, H)_{S}=(X, H)_{x F}$ is an association scheme, which is called the subscheme of $(X, H)$ with respect to $x$ and $F$.

Definition 2.8 Let $(X, H)$ be an association scheme. Let

$$
X / F:=\{x F \mid x \in X\} \quad \text { and } \quad H / / F:=\left\{a^{F} \mid a \in H\right\}
$$

where $a^{F}:=\{(y F, z F) \mid z \in y(F a F)\}$. Then $(X / F, H / / F)$ is an association scheme, which is called the factor scheme of $(X, H)$ over $F$ [10, Section 1.5].

By the definition of the thin residue, for any nontrivial scheme $(X, H),\left(X / \mathbf{O}^{\vartheta}(H), H / /\right.$ $\left.\mathbf{O}^{\vartheta}(H)\right)$ is thin. It is clear that if a factor scheme of $(X, H)$ over $F$ is thin, then $\mathbf{O}^{\vartheta}(H) \subseteq F$. We may suppose that for any closed subset $F$ of $H, F / / \mathbf{O}^{\vartheta}(F)$ (in particular, $H / / \mathbf{O}^{\vartheta}(H)$ ) is a group with respect to the complex product as its group operation (cf. [10, p. 39, p. 41, p. 177]).

\section{Semidirect product}

Let $(X, H)$ and $(Y, K)$ be two schemes. Let $\phi: H \rightarrow H / / \mathbf{O}^{\vartheta}(H)$ be the natural projection map; i.e., $\phi(h)=h^{\mathbf{O}^{\vartheta}(H)}$ for each $h \in H$. Let $\pi: H / / \mathbf{O}^{\vartheta}(H) \rightarrow \operatorname{Aut}(K)$ be a group homomorphism. Then the composite map $\tilde{\pi}:=\pi \circ \phi$ satisfies

$$
\tilde{\pi}(a b)=\tilde{\pi}(a) \tilde{\pi}(b) \quad \text { for all } a, b \in H
$$

Let $x_{0} \in X$ be a fixed point, and let $Z:=\{x y \mid x \in X, y \in Y\}$. (Notice that we use $x y$ instead of $(x, y)$ to denote the points of $Z=X \times Y$.) Let $H \ltimes_{\pi, x_{o}} K:=\{h \cdot k \mid h \in H, k \in K\}$ 
where relations $h \cdot k \subset Z \times Z$ are defined by the rule:

$$
\left(x_{1} y_{1}, x_{2} y_{2}\right) \in h \cdot k \text { if and only if }\left(x_{1}, x_{2}\right) \in h \text { and }\left(y_{1}, y_{2}\right) \in k^{\tilde{\pi}\left(r\left(x_{2}, x_{0}\right)\right)}
$$

for $x_{1} y_{1}, x_{2} y_{2} \in Z$. Then the following lemma guarantees that the pair $\left(Z, H \ltimes_{\pi, x_{0}} K\right)$ satisfies all requirements in Definition 2.1 to be a scheme.

Lemma 3.1 With the above notation, we have:

(i) $H \ltimes_{\pi, x_{0}} K$ is a partition of $Z \times Z$;

(ii) $1_{X} \cdot 1_{Y}$ is the diagonal relation on $Z$;

(iii) For each $h \cdot k \in H \ltimes_{\pi, x_{0}} K$ we have $(h \cdot k)^{*}=h^{*} \cdot\left(k^{*}\right)^{\tilde{\pi}\left(h^{*}\right)}$;

(iv) $\left(Z, H \ltimes_{\pi, x_{0}} K\right.$ ) satisfies Definition 2.1 (iii).

Proof: (i) Suppose the pair $\left(x_{1} y_{1}, x_{2} y_{2}\right)$ belongs to two relations $h \cdot k$ and $h^{\prime} \cdot k^{\prime}$. Then $\left(x_{1}, x_{2}\right) \in h \cap h^{\prime}$ and $\left(y_{1}, y_{2}\right) \in k^{\tilde{\pi}\left(r\left(x_{2}, x_{0}\right)\right)} \cap\left(k^{\prime}\right)^{\tilde{\pi}\left(r\left(x_{2}, x_{0}\right)\right)}$. Since $H$ and $K$ are partitions of $X \times X$ and $Y \times Y$, respectively, we must have $h=h^{\prime}$ and $k=k^{\prime}$. So, each pair belongs to one relation.

Now, for each $\left(x_{1} y_{1}, x_{2} y_{2}\right) \in Z \times Z$, we know that there exist $h:=r\left(x_{1}, x_{2}\right) \in H$ and $k:=r\left(y_{1}, y_{2}\right)^{\tilde{\pi}\left(r\left(x_{0}, x_{2}\right)\right)} \in K$ such that $\left(x_{1} y_{1}, x_{2} y_{2}\right) \in h \cdot k$. That is, $\left(x_{1}, x_{2}\right) \in h$ and $\left(y_{1}, y_{2}\right) \in r\left(y_{1}, y_{2}\right)=k^{\tilde{\pi}\left(r\left(x_{2}, x_{0}\right)\right)}$ since $\tilde{\pi}\left(h h^{*}\right)=\operatorname{id}_{\text {Aut }(K)}$ for each $h \in H$ by the Eq. (3) above. Therefore, $H \ltimes_{\pi, x_{0}} K$ must be a partition of $Z \times Z$.

(ii) Suppose $\left(x_{1} y_{1}, x_{2} y_{2}\right) \in 1_{X} \cdot 1_{Y}$. That is $\left(x_{1}, x_{2}\right) \in 1_{X}$ and $\left(y_{1}, y_{2}\right) \in 1_{Y}{ }^{\tilde{\pi}\left(r\left(x_{2}, x_{0}\right)\right)}$. Since $\tilde{\pi}\left(r\left(x_{2}, x_{0}\right)\right) \in \operatorname{Aut}(K)$, it follows from the Eq. 1 in Section 2 that $1_{Y} \tilde{\pi}\left(r\left(x_{2}, x_{0}\right)\right)=1_{Y}$, and thus, $x_{1}=x_{2}, y_{1}=y_{2}$. Hence we conclude that $1_{X} \cdot 1_{Y}=1_{Z}$.

(iii) If a point $\left(x_{1} y_{1}, x_{2} y_{2}\right)$ belongs to $h \cdot k$, then clearly we have $\left(x_{2}, x_{1}\right) \in h^{*}$ and $\left(y_{2}, y_{1}\right) \in$ $\left(k^{\tilde{\pi}\left(r\left(x_{2}, x_{0}\right)\right)}\right)^{*}$. By the Eqs. (1) and (3), we have

$$
\left(k^{\tilde{\pi}\left(r\left(x_{2}, x_{0}\right)\right)}\right)^{*}=\left(k^{*}\right)^{\tilde{\pi}\left(r\left(x_{2}, x_{0}\right)\right)}=\left(k^{*}\right)^{\tilde{\pi}\left(r\left(x_{2}, x_{1}\right)\right) \tilde{\pi}\left(r\left(x_{1}, x_{0}\right)\right)}=\left(k^{*}\right)^{\tilde{\pi}\left(h^{*}\right) \tilde{\pi}\left(r\left(x_{1}, x_{0}\right)\right)} .
$$

So, we obtain $\left(x_{2} y_{2}, x_{1} y_{1}\right) \in h^{*} \cdot\left(k^{*}\right)^{\tilde{\pi}\left(h^{*}\right)}$ as desired.

(iv) Let $h, h^{\prime} \in H, k, k^{\prime} \in K$ and $\left(x_{1} y_{1}, x_{2} y_{2}\right) \in Z \times Z$, and let

$$
W:=\left\{x_{3} y_{3} \in Z \mid\left(x_{1} y_{1}, x_{3} y_{3}\right) \in h \cdot k,\left(x_{3} y_{3}, x_{2} y_{2}\right) \in h^{\prime} \cdot k^{\prime}\right\} .
$$

Then, for each $x_{3} y_{3} \in W$, we have

$$
\begin{aligned}
& \left(x_{1}, x_{3}\right) \in h \text { and }\left(y_{1}, y_{3}\right) \in k^{\tilde{\pi}\left(r\left(x_{3}, x_{0}\right)\right)}, \text { and } \\
& \left(x_{3}, x_{2}\right) \in h^{\prime} \text { and }\left(y_{3}, y_{2}\right) \in\left(k^{\prime}\right)^{\tilde{\pi}\left(r\left(x_{2}, x_{0}\right)\right)} .
\end{aligned}
$$

Therefore, (iv) follows from the fact that the cardinality of

$$
U:=\left\{y_{3} \mid\left(y_{1}, y_{3}\right) \in k^{\tilde{\pi}\left(r\left(x_{3}, x_{0}\right)\right)},\left(y_{3}, y_{2}\right) \in\left(k^{\prime}\right)^{\tilde{\pi}\left(r\left(x_{2}, x_{0}\right)\right)}\right\}
$$


depends only on $h, h^{\prime}, k, k^{\prime}, r\left(x_{1}, x_{2}\right)$ and $r\left(y_{1}, y_{2}\right)$. Since

$$
\tilde{\pi}\left(r\left(x_{2}, x_{0}\right)\right)=\tilde{\pi}\left(r\left(x_{2}, x_{3}\right)\right) \tilde{\pi}\left(r\left(x_{3}, x_{0}\right)\right),
$$

we have

$$
|U|=p_{k\left(k^{\prime}\right)^{\tilde{\pi}\left(\left(h^{\prime}\right)^{*}\right)}}^{\left(k ^ { \prime \prime } \left(\left(h^{\prime}\right)^{*}\right.\right.}
$$

where

$$
k^{\prime \prime}=r\left(y_{1}, y_{2}\right)^{\tilde{\pi}\left(r\left(x_{0}, x_{2}\right)\right)} \text {. }
$$

Hence, we have

$$
|W|=p_{h h^{\prime}}^{h^{\prime \prime}} p_{k\left(k^{\prime}\right)^{\tilde{\pi}\left(\left(h^{\prime}\right)^{*}\right)}}^{\left(k ^ { \prime \prime } \tilde { \tilde { n } } \left(\left(h^{\prime}\right)^{*}\right.\right.}
$$

where $h^{\prime \prime}=r\left(x_{1}, x_{2}\right)$. Since $\left(k^{\prime \prime}\right)^{\tilde{\pi}\left(r\left(x_{2}, x_{0}\right)\right)}=r\left(y_{1}, y_{2}\right)$ and $h^{\prime \prime}=r\left(x_{1}, x_{2}\right), r\left(x_{1} y_{1}, x_{2} y_{2}\right)=$ $h^{\prime \prime} \cdot k^{\prime \prime}$ holds. Therefore we have

$$
p_{h \cdot k h^{\prime} \cdot k^{\prime}}^{h^{\prime \prime} \cdot k^{\prime \prime}}=p_{h h^{\prime}}^{h^{\prime \prime}} p_{k\left(k^{\prime}\right)^{\tilde{\pi}\left(\left(h^{\prime}\right)^{*}\right)}}^{\left(k^{\prime \prime}\right)}
$$

This completes the proof.

The following lemma asserts that two schemes based on two different fixed points $x_{0}$ and $x_{0}{ }^{\prime}$ are essentially the same.

Lemma 3.2 Let $\left(Z, H \ltimes_{\pi, x_{0}}{ }^{\prime} K\right)$ be the scheme obtained as above by using $x_{0}{ }^{\prime} \in X$ instead of $x_{0}$. Then $\left(Z, H \ltimes_{\pi, x_{0}{ }^{\prime}} K\right)$ is isomorphic to $\left(Z, H \ltimes_{\pi, x_{0}} K\right)$.

Proof: Since $\tilde{\pi}\left(r\left(x_{0}, x_{0}{ }^{\prime}\right)\right) \in \operatorname{Aut}(K)$, the map $\theta$ defined by

$$
\begin{aligned}
\theta: K & \rightarrow K \\
g & \mapsto g^{\tilde{\pi}\left(r\left(x_{0}, x_{0}{ }^{\prime}\right)\right)}
\end{aligned}
$$

gives an automorphism of the scheme $(Y, K)$ together with the identity map on the point set $Y$. Suppose we define a map $\Theta$ between $H \ltimes_{\pi, x_{0}} K$ and $H \ltimes_{\pi, x_{0}} K$ as follows:

$$
\begin{aligned}
\theta: H \ltimes_{\pi, x_{0}} K & \rightarrow H \ltimes_{\pi, x_{0}} K \\
h \cdot_{x_{0}} k & \mapsto h \cdot_{x_{0}{ }^{\prime}} k
\end{aligned}
$$

where

$$
h \cdot x_{0} k=\left\{\left(x_{1} y_{1}, x_{2} y_{2}\right) \in Z \times Z \mid r\left(x_{1}, x_{2}\right)=h, r\left(y_{1}, y_{2}\right)=k^{\tilde{\pi}\left(r\left(x_{2}, x_{0}\right)\right)}\right\}
$$


and

$$
h \cdot x_{0^{\prime}} k=\left\{\left(x_{1} y_{1}, x_{2} y_{2}\right) \in Z \times Z \mid r\left(x_{1}, x_{2}\right)=h, r\left(y_{1}, y_{2}\right)=\theta\left(k^{\tilde{\pi}\left(r\left(x_{2}, x_{0}^{\prime}\right)\right)}\right)\right\}
$$

Then, the pair of maps, $\theta$ defined on $H \ltimes_{\pi, x_{0}} K$ and the identity map on $Z$, yields an isomorphism from $\left(Z, H \ltimes_{\pi, x_{0}} K\right)$ to $\left(Z, H \ltimes_{\pi, x_{0}{ }^{\prime}} K\right)$ as we have

$$
\theta\left(k^{\tilde{\pi}\left(r\left(x_{2}, x_{0}\right)\right)}\right)=k^{\tilde{\pi}\left(r\left(x_{2}, x_{0}\right)\right) \tilde{\pi}\left(r\left(x_{0}, x_{0}^{\prime}\right)\right)}=k^{\tilde{\pi}\left(r\left(x_{2}, x_{0}^{\prime}\right)\right)} .
$$

This completes the proof.

Definition 3.1 The association scheme $\left(Z, H \ltimes_{\pi, x_{0}} K\right)$, or $(X, H) \ltimes_{\pi, x_{0}}(Y, K)$, is called the (external) semidirect product of $(Y, K)$ by $(X, H)$ relative to $\pi$ and $x_{0}$.

Due to the preceding lemma, we will simply write $\left(X \times Y, H \ltimes_{\pi} K\right)$, or $(X, H) \ltimes_{\pi}(Y, K)$, for the semidirect product unless it is necessary to specify the base point. We note that two different homomorphisms $\pi$ and $\pi^{\prime}$ may produce two non-isomorphic schemes $(X \times$ $\left.Y, H \ltimes_{\pi} K\right)$ and $\left(X \times Y, H \ltimes_{\pi^{\prime}} K\right)$ from the same factors $(Y, K)$ and $(X, H)$ as we will see an example later in this section.

The adjacency matrices of the semidirect product can be described in terms of those of factors.

Proposition 3.3 Let $\left(Z, H \ltimes_{\pi} K\right)$ be the semidirect product of $(Y, K)$ by $(X, H)$ relative to $\pi$ and $x_{0}$. Let $\left\{A_{k} \mid k \in K\right\}$ be the set of adjacency matrices of $(Y, K)$. Then the set of adjacency matrices for $\left(Z, H \ltimes_{\pi} K\right)$ is $\left\{A_{h \cdot k} \mid h \cdot k \in H \ltimes_{\pi} K\right\}$, where $A_{h \cdot k}$ is a $|X| \times|X|$ block matrix in which the $\left(x_{i}, x_{j}\right)$-block is given by a $|Y| \times|Y|$ zero-one matrix:

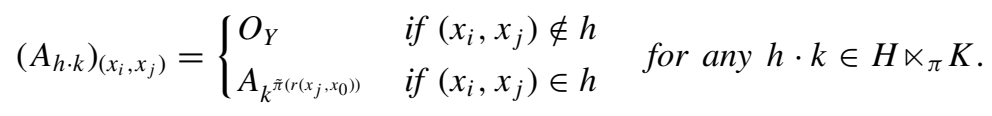

(Here, $O_{Y}$ is the $|Y| \times|Y|$ zero matrix.)

Proof: It follows from the fact that the entry of $A_{h \cdot k}$ is determined by

$$
\left(A_{h \cdot k}\right)_{\left(x_{i} y, x_{j} y^{\prime}\right)}=\delta_{r\left(x_{i} y, x_{j} y^{\prime}\right), h \cdot k}=\delta_{r\left(x_{i}, x_{j}\right), h} \delta_{r\left(y, y^{\prime}\right), k^{\tilde{\pi}\left(r\left(x_{j}, x_{0}\right)\right)}}
$$

where

$$
\delta_{\alpha, \beta}:=\left\{\begin{array}{ll}
1 & \text { if } \alpha=\beta \\
0 & \text { otherwise }
\end{array} .\right.
$$

We now investigate when schemes can be decomposed by their subschemes as semidirect factors; i.e, define an internal semidirect product of schemes. This may be viewed as 
an analog of the decomposition theory over the direct (tensor) product discussed in $[10$, Theorem 2.6.2] and [1, Theorem 3.2].

First, from the definition of the semidirect product $\left(Z, H \ltimes_{\pi} K\right)$, it is easy to see that $H \cdot 1_{Y}:=\left\{h \cdot 1_{Y} \mid h \in H\right\}$ and $1_{X} \cdot K:=\left\{1_{X} \cdot k \mid k \in K\right\}$ are closed subsets of $H \ltimes_{\pi} K$ such that

$$
\left(H \cdot 1_{Y}\right) \cap\left(1_{X} \cdot K\right)=\left\{1_{Z}\right\}
$$

and

$$
\left(H \cdot 1_{Y}\right)\left(1_{X} \cdot K\right)=H \ltimes_{\pi} K .
$$

Moreover, for each $h \in H$ and $k \in K$,

$$
\left|\left(h \cdot 1_{Y}\right)\left(1_{X} \cdot k\right)\right|=\left|\left(h \cdot 1_{Y}\right) *\left(1_{X} \cdot k\right)\left(h \cdot 1_{Y}\right) \cap\left(1_{X} \cdot K\right)\right|=1 .
$$

These properties expressing how the closed subsets sit inside $H \ltimes_{\pi} K$ characterize the structure called an internal semidirect product. To explore this product, we prove the following proposition. The proof of the proposition will follow by a series of lemmas.

Proposition 3.4 Let $(X, G)$ be a scheme. Suppose that there exist $H, K \leq G$ such that

(i) $H \cap K=\left\{1_{X}\right\}$;

(ii) $H K=G$;

(iii) For each $h \in H$ and $k \in K,|h k|=\left|h^{*} k h \cap K\right|=1$.

Then, for each $x \in X,(X, G)$ is isomorphic to the semidirect product of $(X, G)_{x K}$ by $(X, G)_{x H}$ relative to $\pi$ where $\pi$ is uniquely determined by the intersection numbers of $(X, G)$.

Throughout, let $(X, G)$ be a scheme, and let $H, K \leq G$ be two closed subsets satisfying the three conditions given in the hypothesis of Proposition 3.4. We reserve the symbol $\iota$ for the identity element of $\operatorname{Aut}(K)$.

Lemma 3.5 For each $h \in H$, the map $\sigma_{h}: K \rightarrow K$ defined by

$$
k^{\sigma_{h}}:=\text { the unique element of the singleton } \operatorname{set}^{*} k h \cap K
$$

for each $k \in K$, belongs to $\operatorname{Aut}(K)$.

Proof: Since the condition (iii) of Proposition 3.4 holds, for each $k \in K$ and $h \in H$, $k \in k^{\sigma_{h^{*}} \sigma_{h}} \cap k^{\sigma_{h} \sigma_{h^{*}}}$, and $\sigma_{h^{*}} \sigma_{h}=\sigma_{h} \sigma_{h^{*}}=\iota$, for each $h \in H$. Hence $\sigma_{h}$ is well-defined and 
bijective. Moreover, the hypothesis of Proposition 3.4 guarantees that there is a one-to-one correspondence between the sets

$$
\{z \in X \mid(x, z) \in a,(z, y) \in b\}
$$

and

$$
\left\{z^{\prime} \in X \mid\left(x^{\prime}, z^{\prime}\right) \in a^{\sigma_{h}},\left(z^{\prime}, y^{\prime}\right) \in b^{\sigma_{h}}\right\}
$$

where $(x, y) \in c,\left(x^{\prime}, y^{\prime}\right) \in c^{\sigma_{h}}$ with $r\left(x, x^{\prime}\right)=r\left(y, y^{\prime}\right)=h$. It is because, for each element $z$ of the first set, there exists a unique element $z^{\prime}$ such that $z^{\prime} \in z h \cap x^{\prime} K$ due to Proposition 3.4(i)-(ii) and the fact that $H$ and $K$ are closed. Furthermore, $\left(x^{\prime}, z^{\prime}\right) \in a^{\sigma_{h}}$ and $\left(z^{\prime}, y^{\prime}\right) \in b^{\sigma_{h}}$ by Proposition 3.4(iii). Therefore, it is clear that the map $z \mapsto z^{\prime}$ is indeed a bijection. Now the one-to-one correspondence between the two sets implies that $p_{a b}^{c}=p_{a^{\sigma_{h}} b^{\sigma_{h}}}^{c^{\sigma_{h}}}$ for all $a, b, c \in K$ as desired.

Lemma 3.6 For each $t \in \mathbf{O}^{\vartheta}(H), \sigma_{t}=\iota$, the identity permutation of $K$.

Proof: Since $\sigma_{h^{*}} \sigma_{h}=\iota$, it follows from Proposition 3.4(iii) that $k$ is the unique element of $\left(h h^{*}\right)^{*} k\left(h h^{*}\right) \cap K$. This implies that, for each $h \in H$ and $a \in h h^{*}, \sigma_{a}=\iota$. Similarly, we get $\sigma_{b}=\iota$ for each $h_{1}, h_{2} \in H$ and $b \in\left(h_{1} h_{1}^{*}\right)\left(h_{2} h_{2}^{*}\right)$. Thus, the conclusion follows from the definition of $\mathbf{O}^{\vartheta}(H)$ [10, Theorem 2.3.1].

Lemma 3.7 For $h, h^{\prime} \in H$ and $k, k^{\prime} \in K$, if $k^{\prime} \in h k h^{\prime}$, then $h^{\prime}=h^{*}$.

Proof: We have $k^{\prime} \in h k h^{\prime} \cap K \subseteq k^{\sigma_{h^{*}}} h h^{\prime} \cap K$, and thus, $\left(k^{\sigma_{h^{*}}}\right)^{*} k^{\prime} \cap h h^{\prime} \neq \emptyset$. So it follows from Proposition 3.4(i) that $1_{X} \in h^{\prime} h$, or equivalently, $h^{\prime}=h^{*}$.

Lemma 3.8 The map $\sigma: H \rightarrow \operatorname{Aut}(K)\left(h \mapsto \sigma_{h}\right)$ induces the group homomorphism $\pi: H / / \mathbf{O}^{\vartheta}(H) \rightarrow \operatorname{Aut}(K)$ such that $\pi\left(h^{\mathbf{O}^{\vartheta}(H)}\right):=\sigma_{h}$ for each $h \in H$.

Proof: Let $a, b \in H$ with $a^{\mathbf{O}^{\vartheta}(H)}=b^{\mathbf{O}^{\vartheta}(H)}$. Then $a b^{*} \subseteq \mathbf{O}^{\vartheta}(H)$. We first claim that $\{k\}=\left(a b^{*}\right)^{*} k\left(a b^{*}\right) \cap K$ for each $k \in K$. Suppose $k^{\prime} \in\left(a b^{*}\right)^{*} k\left(a b^{*}\right) \cap K$. Then there exist $c \in\left(a b^{*}\right)^{*}$ and $c^{\prime} \in a b^{*}$ such that $k^{\prime} \in c k c^{\prime}$. By Lemma 3.7, $c^{\prime}=c^{*}$, which implies that $k^{\prime}=k^{\sigma_{c^{*}}}$. On the other hand, by Lemma 3.6, $k^{\sigma_{c^{*}}}=k$, so $k^{\prime}=k$, proving the claim. Therefore, we must have $\sigma_{a}=\sigma_{b}$, and thus, $\pi$ is well-defined by Lemma 3.5. Now let $c \in a b$. For each $k \in K, k^{\sigma_{c}}$ is the unique element of $c^{*} k c \cap K$. Notice that

$$
c^{*} k c \cap K \subseteq(a b)^{*} k(a b) \cap K \subseteq b^{*}\left(a^{*} k a\right) b \cap K \subseteq k^{\sigma_{a} \sigma_{b}} b^{*} a^{*} a b \cap K .
$$

With the closed subset $K$ and Proposition 3.4(i), $k^{\sigma_{a} \sigma_{b}} b^{*} a^{*} a b \cap K=\left\{k^{\sigma_{a} \sigma_{b}}\right\}$. This implies that $\sigma_{c}=\sigma_{a} \sigma_{b}$. Hence, $\pi$ is a group homomorphism. 
Lemma 3.9 For any two points $x, z \in X$, there exists a unique pair $\left(z_{1}, z_{2}\right)$ such that $z_{1} \in x H \cap z K$ and $z_{2} \in x K \cap z H$.

Proof: $\quad$ Since $G=H K=K H$, there exists a $\left(z_{1}, z_{2}\right)$ such that $r\left(x, z_{1}\right) \in H, r\left(z_{1}, z\right) \in K$, $r\left(x, z_{2}\right) \in K$ and $r\left(z_{2}, z\right) \in H$. The uniqueness follows from Proposition 3.4(i) and from the fact that $H$ and $K$ are closed.

Proof of Proposition 3.4: Let $\pi$ be the group homomorphism defined as in Lemma 3.8. Consider the semidirect product of $(X, G)_{x K}$ by $(X, G)_{x H}$ relative to $\pi$ and $x$, and the map

$$
\Psi: X \cup G \rightarrow(x H \times x K) \cup\left(H_{x H} \ltimes_{\pi} K_{x K}\right)
$$

defined by

$$
z \in X \mapsto\left(z_{1}, z_{2}\right) \in x H \times x K
$$

and

$$
h k \in G \mapsto h \cdot k,
$$

where $\left(z_{1}, z_{2}\right)$ is the unique pair, $z_{1} \in x H \cap z K$ and $z_{2} \in x K \cap z H$, as seen in Lemma 3.9. Then the uniqueness of $\left(z_{1}, z_{2}\right)$ and the equalities $|X|=|x H||x K|$ and $|G|=|H||K|$ guarantee that $\Psi$ is well-defined and bijective. Therefore, in order to prove that $\Psi$ is an isomorphism, it suffices to show that $r\left(z_{1} z_{2}, w_{1} w_{2}\right)=h \cdot k$ if $r(z, w)=h k$. For given $z$ and $w$, we have a unique element $u \in z H \cap w K$ by Lemma 3.9. Also, $r(z, w)=h k$ implies $u \in z h \cap w k^{*}$. Moreover, since $r\left(z_{1}, z\right) \in r\left(z_{1}, w_{1}\right) r\left(w_{1}, u\right) r(u, z)$ with $r\left(z_{1}, w_{1}\right)$, $r(u, z) \in H$ and $r\left(z_{1}, z\right), r\left(w_{1}, u\right) \in K$, Lemma 3.7 asserts that $r\left(z_{1}, w_{1}\right)=h$. Similarly, we see that $r\left(z_{2}, u\right)=r\left(w_{2}, w\right)=r\left(x, w_{1}\right)$ (cf. figure 1 below). So we have

$$
r\left(z_{2}, w_{2}\right) \in r\left(z_{2}, u\right) r(u, w) r\left(w, w_{2}\right) \cap K=\left\{k^{\sigma_{r\left(w_{1}, x\right)}}\right\}=\left\{k^{\tilde{\pi}\left(r\left(w_{1}, x\right)\right)}\right\},
$$

and conclude that $\left(z_{1} z_{2}, w_{1} w_{2}\right) \in h \cdot k$.

For the remainder of the section, we give a structural characterization of the regular group scheme of dihedral group $D_{2 n}$ of order $2 n$. Then we construct another scheme that is non-isomorphic to the regular group scheme of $D_{2 n}$ but is obtained as the semidirect product of the same factors via a different homomorphism.

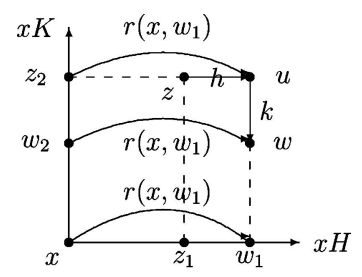

involved.

Figure 1. Relations between the elements involved. 
Every finite group $G$ is associated with a regular group scheme $\mathcal{X}(G)=(G, \tilde{G})$. That is, for given $G$, and $g \in G$, if we define

$$
\tilde{g}:=\left\{(e, f) \in G \times G \mid f^{-1} e=g\right\}
$$

and let $\tilde{G}:=\{\tilde{g} \mid g \in G\}$, then $\mathcal{X}(G)=(G, \tilde{G})$ is a thin scheme. (cf. for example, [2, 3 or 9].) It follows from the definition of the complex product described in the Eq. (2) that $\tilde{G}$ is a group with respect to the complex product as the group operation, with $\tilde{1}$ as identity element. (This operation in the Eq. (2) for thin schemes is often called the complex multiplication.) Moreover, if we consider the relations as group elements and the complex multiplication as a binary operation on the relation set, then the semidirect product of two regular group schemes of $\mathcal{X}(H)$ and $\mathcal{X}(K)$ is isomorphic to the regular group scheme of the semidirect product of two groups $H$ and $K$. We will use relation matrices to describe some schemes below. Recall that the relation matrix of a scheme is a 'colored combination' $\sum_{i} i A_{i}$, of the adjacency matrices $A_{i}$ of the scheme.

Example 3.1 Let $D_{2 n}=\left\langle a, b \mid a^{n}=b^{2}=1, b a=a^{-1} b\right\rangle$ be the dihedral group of order $2 n$. Suppose we denote $\mathcal{X}\left(D_{2 n}\right)=\left(D_{2 n}, \widetilde{D_{2 n}}\right)$ such that its relation set $\widetilde{D_{2 n}}=\left\{\tilde{g}_{i} \mid i=\right.$ $0,1, \ldots, 2 n-1\}$ is defined by, for $x, y \in D_{2 n}$

$$
(x, y) \in \tilde{g_{i}} \text { if and only if } y^{-1} x= \begin{cases}a^{i} & \text { if } 0 \leq i \leq n-1 \\ a^{i-n} b & \text { if } n \leq i \leq 2 n-1\end{cases}
$$

The relation matrix of $\mathcal{X}\left(D_{2 n}\right)$ may be described as follows:

$$
\left(\begin{array}{cc}
C[0,(n-1),(n-2), \ldots, 1] & C[n,(n+1),(n+2), \ldots,(2 n-1)] \\
C[n,(2 n-1),(2 n-2), \ldots,(n+1)] & C[0,1,2, \ldots,(n-2),(n-1)]
\end{array}\right)
$$

where $C\left[c_{1} c_{2} \ldots c_{n}\right]$ denotes the $n \times n$ circulant matrix with $C_{i j}=c_{j-i+1}$ for all $i, j$ with $j-i+1$ being reduced modulo $n$ to a number in $\{1,2, \ldots, n\}$.

Now, let us keep $\mathbb{Z}_{n} \simeq\langle a\rangle$ and $\mathbb{Z}_{2} \simeq\langle b\rangle$ in mind, and consider the regular group schemes $\mathcal{X}\left(\mathbb{Z}_{n}\right)=\left(\mathbb{Z}_{n}, K\right)$ and $\mathcal{X}\left(\mathbb{Z}_{2}\right)=\left(\mathbb{Z}_{2}, H\right)$ with $K=\widetilde{\mathbb{Z}}_{n}$ and $H=\mathbb{Z}_{2}=\{\tilde{0}, \tilde{1}\}$. Here $(x, y) \in \tilde{k} \in K$ if and only if $x-y=k \in \mathbb{Z}_{n}$. Then with the group homomorphism $\pi: H \rightarrow \operatorname{Aut}(K)$ defined by $\pi(\tilde{1})=(\tilde{0})(\tilde{1} n \widetilde{-1})(\tilde{2} n-2) \cdots\left(\widetilde{\left.\frac{n}{2}\right\rfloor} n-\left\lfloor\frac{n}{2}\right\rfloor\right)$, we see that $\mathcal{X}\left(D_{2 n}\right) \simeq \mathcal{X}\left(\mathbb{Z}_{2}\right) \ltimes_{\pi} \mathcal{X}\left(\mathbb{Z}_{n}\right)$ relative to $\pi$ and a base point $b$ due to Propositions 3.3 and 3.4.

So, we have seen that:

The regular group scheme of dihedral group of order $2 n$ is isomorphic to the semidirect product of the regular group scheme of cyclic group of order $n$ by that of cyclic group of order 2 relative to $\pi$. It resembles the situation of groups where the dihedral group of order $2 n$ is the semidirect product of cyclic group of order $n$ by that of order 2 .

As we mentioned earlier, we now illustrate the fact that a different homomorphism may produce a different semidirect product. 
Example 3.2 Suppose we use a trivial homomorphism $\pi^{\prime}$ instead of $\pi$ used in the previous example. Then the relation matrix of $\mathcal{X}\left(\mathbb{Z}_{2}\right) \ltimes_{\pi^{\prime}} \mathcal{X}\left(\mathbb{Z}_{n}\right)$ is

$$
\left(\begin{array}{cc}
C[0,1,2, \ldots,(n-2),(n-1)] & C[n,(n+1),(n+2), \ldots,(2 n-1)] \\
C[n,(n+1),(n+2), \ldots,(2 n-1)] & C[0,1,2, \ldots,(n-2),(n-1)]
\end{array}\right)
$$

Therefore, by comparing this matrix with the relation matrix of $\mathcal{X}\left(D_{2 n}\right)$, it can be verified that the scheme $\mathcal{X}\left(\mathbb{Z}_{2}\right) \ltimes_{\pi^{\prime}} \mathcal{X}\left(\mathbb{Z}_{n}\right)$ is not isomorphic to $\mathcal{X}\left(D_{2 n}\right) \simeq \mathcal{X}\left(\mathbb{Z}_{2}\right) \ltimes_{\pi} \mathcal{X}\left(\mathbb{Z}_{n}\right)$. It is shown that $\mathcal{X}\left(\mathbb{Z}_{2}\right) \ltimes_{\pi^{\prime}} \mathcal{X}\left(\mathbb{Z}_{n}\right)$ is isomorphic to the direct product of $\mathcal{X}\left(\mathbb{Z}_{2}\right)$ and $\mathcal{X}\left(\mathbb{Z}_{n}\right)$.

\section{Application to the classification}

In this section we give a few sporadic examples in order to illustrate the use of the semidirect product in connection with the characterization and classification of association schemes. We shall describe three schemes of order 12 , labelled $\mathcal{X}_{1}, \mathcal{X}_{4}$ and $\mathcal{X}_{6}$ in $[8,(7.12)]$ in terms of the semidirect product of their subschemes. (These schemes are also found in [4 and 5].)

Example 4.1 The relation matrix of $\mathcal{X}_{1}$ of order 12 given in [8, p. 259] is similar to the matrix $\mathcal{R}\left(\mathcal{X}_{1}\right)$ below by the permutation $\left(\begin{array}{lll}2 & 6 & 5\end{array}\right)(811)$ of the corresponding rows and columns:

$$
\mathcal{R}\left(\mathcal{X}_{1}\right)=\left(\begin{array}{llllllllllll}
0 & 2 & 4 & 3 & 5 & 1 & 6 & 7 & 8 & 6 & 7 & 8 \\
4 & 0 & 2 & 1 & 3 & 5 & 8 & 6 & 7 & 8 & 6 & 7 \\
2 & 4 & 0 & 5 & 1 & 3 & 7 & 8 & 6 & 7 & 8 & 6 \\
3 & 5 & 1 & 0 & 2 & 4 & 6 & 7 & 8 & 6 & 7 & 8 \\
1 & 3 & 5 & 4 & 0 & 2 & 8 & 6 & 7 & 8 & 6 & 7 \\
5 & 1 & 3 & 2 & 4 & 0 & 7 & 8 & 6 & 7 & 8 & 6 \\
6 & 8 & 7 & 6 & 8 & 7 & 0 & 4 & 2 & 3 & 1 & 5 \\
7 & 6 & 8 & 7 & 6 & 8 & 2 & 0 & 4 & 5 & 3 & 1 \\
8 & 7 & 6 & 8 & 7 & 6 & 4 & 2 & 0 & 1 & 5 & 3 \\
6 & 8 & 7 & 6 & 8 & 7 & 3 & 1 & 5 & 0 & 4 & 2 \\
7 & 6 & 8 & 7 & 6 & 8 & 5 & 3 & 1 & 2 & 0 & 4 \\
8 & 7 & 6 & 8 & 7 & 6 & 1 & 5 & 3 & 4 & 2 & 0
\end{array}\right) .
$$

Let us denote $\mathcal{X}_{1}$ by $(X, G)$ where $X=\left\{x_{i} \mid 1 \leq i \leq 12\right\}$ and $G=\left\{g_{i} \mid i=0,1, \ldots, 8\right\}$ with $g_{k}:=\left\{\left(x_{i}, x_{j}\right) \mid\left(\mathcal{R}\left(\mathcal{X}_{1}\right)\right)_{(i, j)}=k\right\}$. Then, $\mathcal{X}_{1}$ is shown to be a semidirect product of two subschemes taken as follows. Take two closed subsets $H=\left\{g_{0}, g_{3}, g_{6}\right\}$ and $K=$ $\left\{g_{0}, g_{2}, g_{4}\right\}$. Then it is straightforward to check that $H$ and $K$ satisfy all conditions in Proposition 3.4. So, using the above notation, we have $\mathbf{O}^{\vartheta}(H)=\left\{g_{0}, g_{3}\right\}, H / / \mathbf{O}^{\vartheta}(H)=$ $\left\{g_{0}^{\mathbf{O}^{\vartheta}(H)}=g_{3}^{\mathbf{O}^{\vartheta}(H)}, g_{6}^{\mathbf{O}^{\vartheta}(H)}\right\}$, and $\operatorname{Aut}(K)=\{\iota, \sigma\}$ where $\sigma=\left(g_{2} g_{4}\right)$. Moreover, $\mathcal{X}_{1} \simeq$ $\left(\mathcal{X}_{1}\right)_{x H} \ltimes_{\pi}\left(\mathcal{X}_{1}\right)_{x K}$ where $\pi: H / / \mathbf{O}^{\vartheta}(H) \rightarrow \operatorname{Aut}(K)$ is the homomorphism uniquely determined by $\pi\left(g_{0}^{\mathbf{O}^{\vartheta}(H)}\right)=\pi\left(g_{3}^{\mathbf{O}^{\vartheta}(H)}\right)=\iota$ and $\pi\left(g_{6}^{\mathbf{O}^{\vartheta}(H)}\right)=\sigma$. 
It is easy to see that $\left(\mathcal{X}_{1}\right)_{x H} \simeq \mathcal{X}\left(\mathbb{Z}_{2}\right)_{2} \mathcal{X}\left(\mathbb{Z}_{2}\right)$ and $\left(\mathcal{X}_{1}\right)_{x K} \simeq \mathcal{X}\left(\mathbb{Z}_{3}\right)$ for any point $x \in X$. (For more information on the wreath product '?' we refer to [1 or 7].) Thus, $\mathcal{X}_{1} \simeq\left(\mathcal{X}\left(\mathbb{Z}_{2}\right)<\mathcal{X}\left(\mathbb{Z}_{2}\right)\right) \ltimes_{\pi} \mathcal{X}\left(\mathbb{Z}_{3}\right)$, an external semidirect product.

Example 4.2 The relation matrix of $\mathcal{X}_{4}$ given in $\left[8\right.$, p. 260] is similar to the matrix $\mathcal{R}\left(\mathcal{X}_{4}\right)$ :

$$
\mathcal{R}\left(\mathcal{X}_{4}\right)=\left(\begin{array}{llllllllllll}
0 & 1 & 4 & 4 & 6 & 6 & 2 & 3 & 5 & 5 & 7 & 7 \\
1 & 0 & 4 & 4 & 6 & 6 & 3 & 2 & 5 & 5 & 7 & 7 \\
6 & 6 & 0 & 1 & 4 & 4 & 7 & 7 & 2 & 3 & 5 & 5 \\
6 & 6 & 1 & 0 & 4 & 4 & 7 & 7 & 3 & 2 & 5 & 5 \\
4 & 4 & 6 & 6 & 0 & 1 & 5 & 5 & 7 & 7 & 2 & 3 \\
4 & 4 & 6 & 6 & 1 & 0 & 5 & 5 & 7 & 7 & 3 & 2 \\
2 & 3 & 7 & 7 & 5 & 5 & 0 & 1 & 6 & 6 & 4 & 4 \\
3 & 2 & 7 & 7 & 5 & 5 & 1 & 0 & 6 & 6 & 4 & 4 \\
5 & 5 & 2 & 3 & 7 & 7 & 4 & 4 & 0 & 1 & 6 & 6 \\
5 & 5 & 3 & 2 & 7 & 7 & 4 & 4 & 1 & 0 & 6 & 6 \\
7 & 7 & 5 & 5 & 2 & 3 & 6 & 6 & 4 & 4 & 0 & 1 \\
7 & 7 & 5 & 5 & 3 & 2 & 6 & 6 & 4 & 4 & 1 & 0
\end{array}\right) .
$$

Let us denote $\mathcal{X}_{4}=(X, G)$ where $X=\left\{x_{i} \mid 1 \leq i \leq 12\right\}$ and $G=\left\{g_{i} \mid 0 \leq i \leq 7\right\}$ with each $g_{i}:=\left\{\left(x_{j}, x_{k}\right) \mid\left(\mathcal{R}\left(\mathcal{X}_{4}\right)\right)_{(j, k)}=i\right\}$ and consider two closed subsets $H=$ $\left\{g_{0}, g_{2}\right\}$ and $K=\left\{g_{0}, g_{1}, g_{4}, g_{6}\right\}$ of $G$. Then, we have $\mathbf{O}^{\vartheta}(H)=\left\{g_{0}\right\}$ and $H / / \mathbf{O}^{\vartheta}(H)=$ $\left\{g_{0}^{\mathbf{O}^{\vartheta}(H)}, g_{2}^{\mathbf{O}^{\vartheta}(H)}\right\}$. We note that $\left(\mathcal{X}_{4}\right)_{x H} \simeq \mathcal{X}\left(\mathbb{Z}_{2}\right)$ and $\left(\mathcal{X}_{4}\right)_{x K} \simeq \mathcal{X}\left(\mathbb{Z}_{2}\right)<\mathcal{X}\left(\mathbb{Z}_{3}\right)$. By Proposition 3.4, we have $\mathcal{X}_{4} \simeq \mathcal{X}\left(\mathbb{Z}_{2}\right) \ltimes_{\pi}\left(\mathcal{X}\left(\mathbb{Z}_{2}\right)<\mathcal{X}\left(\mathbb{Z}_{3}\right)\right)$ where $\pi: H / / \mathbf{O}^{\vartheta}(H) \rightarrow \operatorname{Aut}(K)$ is the homomorphism uniquely determined as $\pi\left(g_{0}^{\mathbf{O}^{\vartheta}(H)}\right)=\iota$ and $\pi\left(g_{2}^{\mathbf{O}^{\vartheta}(H)}\right)=\left(g_{0}\right)\left(g_{1}\right)\left(g_{4} g_{6}\right)$.

Example 4.3 The relation matrix of $\mathcal{X}_{6}$ given in [8, p. 261] is similar to $\mathcal{R}\left(\mathcal{X}_{6}\right)$ :

$$
\mathcal{R}\left(\mathcal{X}_{6}\right)=\left(\begin{array}{cccccccccccc}
0 & 1 & 2 & 6 & 6 & 6 & 3 & 5 & 4 & 7 & 7 & 7 \\
2 & 0 & 1 & 6 & 6 & 6 & 4 & 3 & 5 & 7 & 7 & 7 \\
1 & 2 & 0 & 6 & 6 & 6 & 5 & 4 & 3 & 7 & 7 & 7 \\
6 & 6 & 6 & 0 & 1 & 2 & 7 & 7 & 7 & 3 & 5 & 4 \\
6 & 6 & 6 & 2 & 0 & 1 & 7 & 7 & 7 & 4 & 3 & 5 \\
6 & 6 & 6 & 1 & 2 & 0 & 7 & 7 & 7 & 5 & 4 & 3 \\
3 & 4 & 5 & 7 & 7 & 7 & 0 & 2 & 1 & 6 & 6 & 6 \\
5 & 3 & 4 & 7 & 7 & 7 & 1 & 0 & 2 & 6 & 6 & 6 \\
4 & 5 & 3 & 7 & 7 & 7 & 2 & 1 & 0 & 6 & 6 & 6 \\
7 & 7 & 7 & 3 & 4 & 5 & 6 & 6 & 6 & 0 & 2 & 1 \\
7 & 7 & 7 & 5 & 3 & 4 & 6 & 6 & 6 & 1 & 0 & 2 \\
7 & 7 & 7 & 4 & 5 & 3 & 6 & 6 & 6 & 2 & 1 & 0
\end{array}\right) .
$$


By denoting the relation set of $\mathcal{X}_{6}$ by $G=\left\{g_{i} \mid 0 \leq i \leq 7\right\}$ as before, and taking $H=\left\{g_{0}, g_{3}\right\}$ and $K=\left\{g_{0}, g_{1}, g_{2}, g_{6}\right\}$, we have $\left(\mathcal{X}_{6}\right)_{x H} \simeq \mathcal{X}\left(\mathbb{Z}_{2}\right)$ and $\left(\mathcal{X}_{6}\right)_{x K} \simeq \mathcal{X}\left(\mathbb{Z}_{3}\right)_{2} \mathcal{X}\left(\mathbb{Z}_{2}\right)$ for any point $x \in X$. By Proposition 3.4, we see that $\mathcal{X}_{6} \simeq \mathcal{X}\left(\mathbb{Z}_{2}\right) \ltimes_{\pi}\left(\mathcal{X}\left(\mathbb{Z}_{3}\right)<\mathcal{X}\left(\mathbb{Z}_{2}\right)\right)$ where the homomorphism $\pi: H / / \mathbf{O}^{\vartheta}(H) \rightarrow$ Aut $(K)$ is uniquely determined as $\pi\left(g_{0}^{\mathbf{O}^{\vartheta}(H)}\right)=\iota$ and $\pi\left(g_{3}^{\mathbf{O}^{\vartheta}(H)}\right)=\left(g_{0}\right)\left(g_{6}\right)\left(g_{1} g_{2}\right)$.

Finally, we close the section with some remarks.

Remark 4.4 (i) Another definitions of semidirect products were given in [6 and 10] as we mentioned in Introduction.

The Zieschang's semidirect product may be described as follows. Let $(Y, K)$ be an association scheme and $H$ be an arbitrary group. We say that ' $H$ acts on $(Y, K)$ ' if there exists a homomorphism $\theta: H \rightarrow \operatorname{Aut}(Y, K)$. We shall write $y^{h}$ instead of $y^{\theta(h)}$. The semidirect product defined in [10] has $H \times Y$ as the set of points. The relations are parameterized by pairs $(h, k) \in H \times K$, and the relation $(\widetilde{h, k})$ corresponding to a pair $(h, k)$ is defined by $\left\{\left(h_{1} y_{1}, h_{2} y_{2}\right) \mid\left(y_{1}^{h_{1}}, y_{2}\right) \in k, h_{1}^{-1} h_{2}=h\right\}$. Then it is shown that $\{(\widetilde{h, k}) \mid(h, k) \in H \times K\}$ forms an association scheme.

The Muzychuk's semidirect product of $(Y, K)$ by $H$ given in [6] may be described as follows. We say that ' $H$ acts on $K$ ' if there exists a homomorphism $\pi: H \rightarrow \operatorname{Aut}(K)$. We shall again write $k^{h}$ instead of $k^{\pi(h)}$. For each pair $(h, k) \in H \times K$, a relation $h \ltimes k$ on the set $H \times Y$ is defined in the following way: $h \ltimes k:=\left\{\left(h_{1} y_{1}, h_{2} y_{2}\right) \mid\left(y_{1}, y_{2}\right) \in k^{h_{2}^{-1}}, h_{1}^{-1} h_{2}=h\right\}$. In [6], Muzychuk has shown that the Zieschang's definition is a particular case of his; i.e., given the group homomorphism $\theta: H \rightarrow \operatorname{Aut}(Y, K)$, if the action of $H$ on $K$ is defined by the rule: $k^{h}=h^{-1} k h$, then two association schemes given by $\{h \ltimes k \mid(h, k) \in H \times K\}$ and $\{(\widetilde{h, k}) \mid(h, k) \in H \times K\}$ are isomorphic.

(ii) We now see that Muzychuk's product is, in turn, a particular case of the one defined in this paper. For a given thin factor $(X, H)$, in order to obtain the Muzychuk's product, we only need to take the base point $x_{0}$ as the point corresponding to the identity element of the associated group to the thin scheme $(X, H)$ (cf. [10, p. 177 Theorem A(iii)]). Thus the semidirect product defined in this paper is a natural extension of the semidirect products defined in [6 and 10].

We also note that the semidirect product $\mathcal{X}\left(\mathbb{Z}_{2}\right) \ltimes \mathcal{X}\left(P_{5}\right)$, where $\mathcal{X}\left(P_{5}\right)$ is the scheme whose first relation graph is the pentagon, is the scheme of the smallest order that can not be constructed by the semidirect product defined in [6 and 10]. Among the three schemes of order 12 which are illustrated above, the scheme $\mathcal{X}_{1}$ in Example 4.1 is not obtained by Muzychuk's construction either.

(iii) We have seen that $\mathcal{X}\left(D_{2 n}\right)$ can be described as the semidirect product of $\mathcal{X}\left(\mathbb{Z}_{n}\right)$ by $\mathcal{X}\left(\mathbb{Z}_{2}\right)$. However, it is not possible to describe $\mathcal{X}\left(D_{2 n}\right)$ by the direct product or wreath product of any proper subschemes (See [1, p. 7] and [1, Theorem 3.2]).

The direct product and wreath product operation can produce one or two schemes from a given pair. However, the semidirect product may produce many schemes from a pair of schemes; in fact, the number is depending on the number of nontrivial homomorphisms $\pi$ available. 
(vi) This work is a continuation of our efforts to characterize schemes in terms of 'smaller' and more 'basic' schemes via some sorts of product operations and/or fusion and fission processes. Propositions 3.3 and 3.4 are perhaps useful in classification as well as the development of the theory. However, there are still many schemes that we do not have such an interpretation (characterization) in terms of known product operations including the semidirect product. For example, there are still nine schemes of order 12 that we do not know whether they are decomposable by any products. The nine schemes are labelled with $\mathcal{X}_{i}$, $i \in\{2,3,5,7,8,9,10,11\}$ and $\mathcal{Y}$ in $[8,7.12]$. We have more and more of such schemes as the order of schemes gets larger.

\section{Acknowledgment}

The authors are indebted to Dr. M. Muzychuck and Dr. P.-H. Zieschang for part of the result reported in this paper. This work has grown out from a conversation of the second author with them on a possible generalization of the concept of the semidirect product introduced in Section 2.7 of [10]. We thank the referees for valuable suggestions and remarks. The third author also thanks the Mathematics Department at POSTECH for the warm hospitality during his visit at which this research was carried out.

\section{References}

1. S. Bang and S.Y. Song, "Characterization of maximal rational circulant association schemes," in Codes and Designs, K.T. Arasu and A. Seress (Eds.), Ohio State Univ. Math. Inst. Publ. 10, Walter de Gruyter, Berlin, 2002, pp. 37-48.

2. E. Bannai and T. Ito, Algebraic Combinatorics I: Association Schemes, Benjamin/Cummings, Menlo Park, CA, 1984.

3. P.J. Cameron, Permutation Groups, Cambridge University Press, Cambridge, UK, 1999.

4. A. Hanaki and I. Miyamoto, "Classification of primitive association schemes of order up to 22," Kyushu J. Math. 54(1) (2000), 81-86. (cf. http://kissme.shinshu-u.ac.jp/as/)

5. M. Hirasaka, "The classification of association schemes with 11 or 12 vertices," Kyushu J. Math. 51 (1997), 413-428.

6. M. Muzychuk, Semidirect product, a short note, March 22, 2000.

7. M. Muychuk, M. Klin, and R. Pöschel, "The isomorphism problem for circulant graphs via Schur ring theory," DIMACS Series in Discrete Mathematics 56 (2001), 241-264.

8. K. See and S. Y. Song, "Association schemes of small order," J. Statist. Plann. Inference 73 (1998), $225-271$.

9. H. Wielandt, Finite Permutation Groups, Academic Press, New York, 1964.

10. P.-H. Zieschang, An Algebraic Approach to Association Schemes, Lecture Notes in Mathematics, Vol. 1628, Springer, 1996. 